\title{
DEGUM-Promotionspreis 2018
}

Die Deutsche Gesellschaft für Ultraschall in der Medizin (DEGUM) vergibt einen Preis für eine hervorragende Dissertation auf dem Gebiet des medizinischen Ultraschalls. Der Promotionspreis ist mit 2000 Euro dotiert und kann geteilt werden.

Für den DEGUM-Promotionspreis können Dissertationen berücksichtigt werden, die nach dem 30. Juni 2015 von einer deutschen Medizinischen Fakultät angenommen wurden. Die Arbeit sollte in der Regel publiziert oder zur Publikation angenommen sein. Hierbei sollte der Bewerber als Erstautor oder als geteilter Erstautor gelistet sein. Die Zustimmung des Betreuers der Arbeit zur Bewerbung wird vorausgesetzt.
Die Bewerbung ist auf elektronischem Weg bis zum 31. Mai 2018 an die DEGUM-Geschäftsstelle (geschaeftsstelle@degum.de) zu richten. Der Bewerbung sind in digitaler Form (PDF-Datei) und ebenfalls auf elektronischem Weg die Dissertation und, sofern verfügbar, die akzeptierte Publikation beizufügen. Des Weiteren ist vom Antragsteller eine formlose Erklärung beizulegen, dass die vorgelegte(n) Arbeit(en) für keinen andersartigen Preis eingereicht wurde(n).

Der Wissenschaftsbeirat der DEGUM bewertet die Arbeit auf der Grundlage von 2 Gutachten und entscheidet über die Preisvergabe; seine Entscheidung ist endgültig und nicht anfechtbar.
Die Preisverleihung erfolgt während des Dreiländertreffens 2018 in Basel/Schweiz (14. - 16. November 2018). Die persönliche Anwesenheit der Preisträgerin/des Preisträgers ist obligat. Von ihr/ihm wird erwartet, dass sie/er die mit dem DEGUM-Preis ausgezeichnete Arbeit während des Dreiländertreffens in Form eines Vortrags vorstellt.

PD Dr. med. Kai-Sven Heling, Präsident der DEGUM

Prof. Dr. med. A. Hagendorff, Sekretär der DEGUM 